\title{
Technique and Interpretation of Arthrogram for Developmental Dysplasia of the Hip
}

\author{
Mandar V. Agashe ${ }^{1}$ [D $\cdot$ Darshan Kapoor ${ }^{1}$ - Sandeep V. Vaidya ${ }^{2}$
}

Received: 29 July 2021 / Accepted: 13 August 2021 / Published online: 17 November 2021

(c) Indian Orthopaedics Association 2021

\begin{abstract}
Closed reduction, arthrography and hip spica application is the treatment of choice for DDH between 6 and 18 months. There is a lot of controversy about what exactly constitutes an "acceptable" closed reduction and arthrogram and the arthrography findings are often difficult to interpret. In this video, the authors describe the technique to perform the arthrogram as well as the interpretation of the same. Various static parameters such as the femoral head coverage, the medial dye pool and the hourglass constriction are shown. In addition, the validity of the safe zone is discussed. With this video technique article, the authors hope that it becomes easy for the budding paediatric orthopaedic surgeon to perform and interpret the DDH arthrogram with ease.
\end{abstract}

Supplementary Information The online version contains supplementary material available at https://doi.org/10.1007/s43465-021-00493-4.

Author Contributions MVA, SVV: Concepts, MVA, DK, SVV: Design, MVA, SVV: Definition of intellectual content, MVA, DK: Literature search, MVA, SVV: Clinical studies, Data acquisition, Data analysis, MVA: Statistical analysis, MVA, DK: Manuscript preparation, MVA, DK, SVV: Manuscript editing, Manuscript review, MVA: Guarantor.

\section{Declarations}

Conflict of interest (If present, give more details): nil.

Ethical Standard Statement This article does not contain any studies with human or animal subjects performed by the any of the authors.
Informed Consent For this type of study informed consent is not required.

Source(s) of Support Nil.

Presentation at a Meeting Nil.

Publisher's Note Springer Nature remains neutral with regard to jurisdictional claims in published maps and institutional affiliations.

Mandar V. Agashe

mandarortho@gmail.com

Darshan Kapoor

dr.dmkapoor@gmail.com

Sandeep V. Vaidya

drsvvaidya@gmail.com

1 Agashe's Paediatric SuperSpeciality Clinic, 16094, Kohinoor

City Phase 2, Kirol Road, Kurla West, Mumbai 400070,

India

2 Pinnacle Ortho Centre, Thane, India 